\title{
Efficient transceiver design for large-scale antenna systems
}

Joonwoo Shin

\begin{abstract}
We present linear transceiver design strategies for large-scale multiple-input multiple-output downlink systems when the user nodes have multiple receive antennas, and they receive multiple streams. First, we propose a matched-filtering-based optimal power allocation method using the sum-rate maximization criterion and utilizing the favorable propagation property of large-scale antenna systems. In uncorrelated transmit antennas, we observe that the effective channel can be represented by the receive antenna correlation matrix. Motivated by this observation, we also propose a suboptimal two-step power allocation method in which each user node is equipped with two receive antennas to reduce the computational complexity of the optimum power allocation method. Compared with the optimum power allocation method, the proposed two-step method reduces the dimensions of the bisection search space while achieving essentially the same level of sum rate.
\end{abstract}

Keywords: Large-scale antenna systems; Multiple-input multiple-output (MIMO); Power allocation; Sum-rate maximization

\section{Introduction}

Large-scale antenna or massive multiple-input multipleoutput (MIMO) system, a system defined as a large number of antenna-equipped base station (BS) while serving a large number of users simultaneously has been identified as a promising air interface technology [1-3]. A salient feature of large-scale antenna systems is that, when the number of BS antennas grows large, the random channel vectors between the users and the BS become pairwisely orthogonal [4]. Therefore, in the limit of infinite number of antennas, with simple linear processing approaches such as a matched filter precoder, inter-user interferences (IUI) disappear completely [5]. The IUI elimination feature of large-scale antenna systems has sparked a flurry of research activities aimed at understanding the signal processing, information theoretic ramifications, and realistic channel behavior of massive MIMO sytems [1-4,6-8]. In $[1,6]$, large-scale antenna systems are reviewed from various perspectives including antenna and propagation aspects, transceiver design, and fundamental information theoretic gains. A follow-up tutorial [2,3] briefly discussed recent works. To the best of our knowledge, most of works

Correspondence: joonoos@kmou.ac.kr

Division of Navigation Science, Korea Maritime and Ocean University (KMOU), Dongsam-dong, Youngdo-gu, 606-791 Busan, Republic of Korea on the large-scale antenna systems assume that user terminals are equipped with a single receive antenna $[1,5,9]$.

In this paper, we aim to design transceivers for the large-scale antenna system where multiple streams are designated for user terminals with multiple receive antennas. Here, we assume that the number of BS antennas exceeds the total number of antennas of users. Inspired by the fact that in large-scale antenna systems a simple matched-filtering precoder can eliminate the IUI effectively $[1,5]$, we adopt conjugate beamforming as the first part of the precoder. After that, a sum-rate maximizing processing is appended. We observe that when the spacing between transmit antennas is sufficiently large, sum-rate maximizing transceivers can be obtained with the receiver correlation matrices. Motivated by this observation, when each terminal has two receive antennas, we propose a suboptimal two-step power allocation method. In general, the search space dimension of the optimal power allocation algorithm is proportional to the number of users and the number of streams per user. With the proposed two-step power allocation method, the search space size is only proportional to the number of users. Through numerical simulations, we verify that the sum-rate performance of the proposed methods outperform the equal power allocation method. In addition, we demonstrate the

\section{望 Springer}


effectiveness of the proposed two-step power allocation method.

The following notations are used. We employ uppercase boldface letters for matrices and lowercase boldface letters for vectors. For any general matrix $\mathbf{X}, \mathbf{X}^{T}, \mathbf{X}^{H}, \operatorname{Tr}(\mathbf{X})$, $\operatorname{SVD}(\mathbf{X})$, and $[\mathbf{X}]_{i, j}$ denote the transpose, Hermitian transpose, trace, singular value decomposition, and the $(i, j)$-th element of $\mathbf{X}$, respectively. An identity matrix of size $n$ is denoted by $\mathbf{I}_{n}$.

\section{System model and preliminaries}

\subsection{MU-MIMO system model}

We consider the time-division-duplexing (TDD) downlink single-cell MU-MIMO systems. The system includes one base station with $N_{t}$ transmit antennas that transmits data streams to $K$ user nodes. Each user terminal has multiple receive antennas. Without loss of generality, we assume that each user node has $N_{r}$ receive antennas. Furthermore, from the TDD channel reciprocity between the downlink and uplink, we assume that the downlink channel state information is known to the transmit side; and due to the single-cell assumption, we do not consider the pilot contamination problems [10]. The information signal vector for the $k$-th user node is denoted by $\mathbf{s}_{k} \in \mathcal{C}^{N_{r} \times 1}$ and its power is normalized as $\mathbb{E}\left(\mathbf{s}_{k} \mathbf{s}_{k}^{H}\right)=\mathbf{I}_{N_{r}}$. The signal $\mathbf{s}_{k}$ is linearly precoded by the precoding matrix $\mathbf{T}_{k} \in$ $\mathcal{C}^{N_{t} \times N_{r}}$. The precoded signal vector $\mathbf{x}_{k}=\mathbf{T}_{k} \mathbf{s}_{k}, 1 \leq k \leq K$ is transmitted to the $k$-th user node from the base station. Then, the received signal vector at the $k$-th user node can be represented by

$$
\mathbf{y}_{k}=\sqrt{g_{k}} \mathbf{H}_{k} \mathbf{x}_{k}+\sqrt{g_{k}} \mathbf{H}_{k} \sum_{i \neq k}^{K} \mathbf{x}_{i}+\mathbf{n}_{k}
$$

where $\mathbf{H}_{k} \in \mathcal{C}^{N_{r} \times N_{t}}$ is the MIMO channel from the base station to the $k$-th user node and $\mathbf{n}_{k}$ equals the additive white Gaussian noise (AWGN) vector with zero mean and $\mathbb{E}\left[\mathbf{n}_{k} \mathbf{n}_{k}^{H}\right]=\sigma_{n}^{2} \mathbf{I}_{N_{r}}$. The parameter $g_{k}$ models the geometric attenuation and shadow fading, which is assumed to be independent over transmit antenna and to be constant over many coherence time intervals.

To introduce the channel correlation, the Kronecker model is adopted to describe $\mathbf{H}_{k}$ as [11]:

$$
\mathbf{H}_{k}=\mathbf{R}_{r_{k}}^{1 / 2} \mathbf{H}_{w_{k}} \mathbf{R}_{t_{k}}^{1 / 2}
$$

where $\mathbf{R}_{r_{k}} \in \mathcal{C}^{N_{r} \times N_{r}}$ and $\mathbf{R}_{t_{k}} \in \mathcal{C}^{N_{t} \times N_{t}}$ are the receive correlation matrix and the transmit correlation matrix of the $k$-th user node, respectively. The $(i, j)$-th elements of $\mathbf{R}_{r_{k}}$ and $\mathbf{R}_{t_{k}}$ are defined as $\left[\mathbf{R}_{r_{k}}\right]_{i, j}=\rho_{r_{k}}^{|i-j|}$ and $\left[\mathbf{R}_{t_{k}}\right]_{i, j}=\rho_{t_{k}}^{|i-j|}$, $\left(0 \leq \rho_{t_{k}}, \rho_{t_{k}} \leq 1\right)$, respectively, assuming the uniform linear array antenna sets. When antenna spacing at the transmit side is sufficiently large, we can assume that the columns of $\mathbf{H}_{k}$ are independent ${ }^{\mathrm{a}}$, i.e., $\mathbf{R}_{t_{k}}=\mathbf{I}_{N_{t}}, \forall k$. On the other hand, the receive antennas of the user terminal are closely spaced due to the size limitation of the user terminal. Therefore, we assume that the rows of $\mathbf{H}_{k}$ 's are correlated, i.e., $\mathbf{R}_{r_{k}} \neq \mathbf{I}_{N_{r}}, \forall k$. The elements of $\mathbf{H}_{w_{k}}$ are circularly symmetric complex Gaussian random variables with zero mean and unit variance, and they have an independent and identically distribution. Then, the user node combines its received signal with decoding matrix $\mathbf{D}_{k} \in \mathcal{C}^{N_{r} \times N_{r}}$ to decode the desired signals:

$$
\hat{\mathbf{s}}_{k}=\sqrt{g_{k}} \mathbf{D}_{k} \mathbf{H}_{k} \mathbf{x}_{k}+\sqrt{g_{k}} \mathbf{D}_{k} \mathbf{H}_{k} \sum_{i \neq k}^{K} \mathbf{x}_{k}+\mathbf{D}_{k} \mathbf{n}_{k}
$$

\subsection{Review of results on the large-scale MIMO systems}

Lemma 1. Let $\mathbf{a}, \mathbf{b} \in \mathcal{C}^{N_{t} \times 1}$ be two independent vectors with distribution $\mathcal{C N}\left(0, c \mathbf{I}_{N_{t}}\right)$, where $c$ is an arbitrary constant. Then,

$$
\lim _{N_{t} \rightarrow \infty} \frac{\mathbf{a}^{H} \mathbf{b}}{N_{t}} \stackrel{\text { a.s. }}{=} 0 \quad \text { and } \quad \lim _{N_{t} \rightarrow \infty} \frac{\mathbf{a}^{H} \mathbf{a}}{N_{t}} \stackrel{\text { a.s. }}{=} c
$$

where $\stackrel{a . s .}{=}$ denotes the almost sure convergence. Lemma 1 is called the favorable propagation (FP) property of large-scale antenna systems [1].

Due to lemma 1, a matched-filtering precoder is popularly adopted for the large-scale MIMO precoding method due to its computational efficiency and rate achievability $[1,5]$. We assume $\mathbf{T}_{k}=\frac{1}{N_{t}} \mathbf{H}_{k}^{H} \mathbf{M}_{k}$ and the precoding matrix $\mathbf{M}_{k} \in \mathcal{C}^{N_{r} \times N_{r}}$ after matched-filtering. Then, the decoded signal $\hat{\mathbf{s}}_{k}$ can be rewritten as

$$
\hat{\mathbf{s}}_{k}=\frac{\sqrt{g_{k}}}{N_{t}} \mathbf{D}_{k} \mathbf{H}_{k} \mathbf{H}_{k}^{H} \mathbf{M}_{k} \mathbf{s}_{k}+\frac{\sqrt{g_{k}}}{N_{t}} \mathbf{D}_{k} \mathbf{H}_{k} \sum_{i \neq k}^{K} \mathbf{H}_{i}^{H} \mathbf{M}_{i} \mathbf{s}_{i}+\mathbf{D}_{k} \mathbf{n}_{k}
$$

Different from the case for when user terminals are equipped with single receive antenna, the power normalization factor is not included in Equation 3. Instead, the precoding matrices $\mathbf{M}_{k}$ 's are designed to satisfy the transmit power constraint, $\sum_{k=1}^{K} \operatorname{Tr}\left(\mathbf{T}_{k} \mathbf{T}_{k}^{H}\right) \leq P_{t}$, and $P_{t}$ is the maximum transmission power of base station.

\section{Sum-rate maximizing transceiver design}

According to lemma 1 , when $N_{t} \rightarrow \infty$, we have

$$
\frac{1}{N_{t}} \mathbf{H}_{w_{i}} \mathbf{H}_{w_{i}}^{H} \cong \mathbf{I}_{N_{r}} \quad \text { and } \quad \frac{1}{N_{t}} \mathbf{H}_{w_{i}} \mathbf{H}_{w_{j}}^{H} \cong \mathbf{0}_{N_{r}} \quad(i \neq j)
$$

Recent channel measurement campaigns have shown that multi-user MIMO systems with large antenna arrays have characteristics that satisfy the above assumption 
(Equation 4) fairly well [7]. Then, we have the decoded signal being free of inter-user interference as follows:

$$
\hat{\mathbf{s}}_{k} \cong \sqrt{g_{k}} \mathbf{D}_{k} \mathbf{R}_{r_{k}} \mathbf{M}_{k} \mathbf{s}_{k}+\mathbf{D}_{k} \mathbf{n}_{k}
$$

Here, we observe that the remaining sum-rate maximizing processing $\left\{\mathbf{D}_{k}\right\}$ and $\left\{\mathbf{M}_{k}\right\}$ can be found by considering receive antenna correlation matrix $\left\{\mathbf{R}_{r_{k}}\right\}$. From channel diagonalization $\operatorname{SVD}\left(\mathbf{R}_{r_{k}}\right)=\mathbf{V}_{k} \Lambda_{k} \mathbf{V}_{k}^{H}$, we have $\mathbf{M}_{k}=$ $\mathbf{V}_{k} \Gamma_{k}^{1 / 2}$ and $\mathbf{D}_{k}=\mathbf{V}_{k}^{H}$, where $\Gamma_{k} \in \mathcal{R}^{N_{r} \times N_{r}}$ is the power loading diagonal matrix. The information rate ${ }^{\mathrm{b}}$ for the $k$-th user node can be represented as

$$
\mathrm{R}_{k}=\log \left\{\operatorname{det}\left(\mathbf{I}_{N_{r}}+\sigma_{n}^{-2} g_{k} \Lambda_{k}^{2} \Gamma_{k}\right)\right\}
$$

The sum-rate maximizing power allocation matrices $\Gamma_{k}$ s are calculated by solving the following optimization problem,

$$
\begin{aligned}
\left\{\Gamma_{k, \mathrm{opt}}\right\}=\arg _{\left\{\Gamma_{k}\right\}} \max \sum_{k=1}^{K} \mathrm{R}_{k} \\
\quad \text { subject to } \sum_{k=1}^{K} \operatorname{Tr}\left(\Lambda_{k} \Gamma_{k}\right) \leq N_{t} P_{t}
\end{aligned}
$$

The power constraint of Equation 7 is the average power constraint that is obtained by

$$
\begin{aligned}
\sum_{k} \mathbb{E}\left[\operatorname{Tr}\left(\mathbf{T}_{k} \mathbf{T}_{k}^{H}\right)\right] & =\sum_{k} \mathbb{E}\left[\operatorname{Tr}\left(\mathbf{R}_{r_{k}}^{1 / 2} \mathbf{H}_{w_{k}} \mathbf{H}_{w_{k}}^{H} \mathbf{R}_{r_{k}}^{1 / 2} \mathbf{V}_{k} \Gamma_{k} \mathbf{V}_{k}^{H}\right)\right] \\
& =\frac{1}{N_{t}} \sum_{k} \operatorname{Tr}\left[\Lambda_{k} \Gamma_{k}\right] \leq P_{t}
\end{aligned}
$$

The solution of the problem (Equation 7) is the well-known water-filling solution that requires numerical bisection search [12]. Finally, we find the sum-rate maximizing transceivers for large-scale antenna systems $\mathbf{T}_{k}^{(\text {opt })}=\mathbf{H}_{k}^{H} \mathbf{V}_{k} \Gamma_{k, \text { opt }}^{1 / 2}$ and $\mathbf{D}_{k}=\mathbf{V}_{k}^{H}, \forall k$.

\section{Two-step power allocation}

The proposed optimum precoder in Section 33 requires a bisection search to optimally allocate the transmit power. In this section, we propose a computationally efficient power allocation method when each user node has two receive antennas ${ }^{\mathrm{c}}$, i.e., $N_{r}=2$.

To derive the two-step power allocation method, we temporarily assume a high SNR regime in which the equal power allocation $\Gamma_{k}=\gamma_{k} \mathbf{I}_{N_{r}}$ for all $k$ is the optimum and there is no receive antenna correlation, i.e., $\rho_{r_{k}}=0$ or $\Lambda_{k}=\mathbf{I}_{N_{r}}$ for all $k$. Then, the optimization problem (Equation 7) can be represented as

$$
\begin{aligned}
& \max _{\left\{\gamma_{k}\right\}} \sum_{k=1}^{K} \log \left\{\operatorname{det}\left(\mathbf{I}_{N_{r}}+\sigma_{n}^{-2} g_{k} \gamma_{k} \mathbf{I}_{N_{r}}\right)\right\} \\
& \text { subject to } \sum_{k=1}^{K} \gamma_{k} \leq \frac{N_{t} P_{t}}{N_{r}}
\end{aligned}
$$

where the power constraint is obtained by $\sum_{k} \operatorname{Tr}\left(\Lambda_{k} \Gamma_{k}\right)=\sum_{k} \operatorname{Tr}\left(\gamma_{k} \mathbf{I}_{N_{r}}\right)=\sum_{k} N_{r} \gamma_{k}$. The power loading parameters $\left\{\gamma_{k}\right\}$ can be found by the water-filling algorithm. Note that compared with the optimum power allocation calculation (Equation 7), the search space of Equation 8 is reduced to $K$ from $K N_{r}$. Through the first step, we find the amount of per user power allocation.

Now, with per user power constraint $\left\{\hat{p}_{k}\right\}$ where $\hat{p}_{k}=$ $N_{r} \gamma_{k}$, the optimization problem (Equation 7) is divided into $K$ subproblems. The $k$-th subproblem is represented by

$$
\begin{gathered}
\arg _{\hat{\Gamma}_{k}} \max \log \left\{\operatorname{det}\left(\mathbf{I}_{N_{r}}+\sigma_{n}^{-2} g_{k} \Lambda_{k}^{2} \hat{\Gamma}_{k}\right)\right\} \\
\text { subject to } \operatorname{Tr}\left(\Lambda_{k} \hat{\Gamma}_{k}\right) \leq \hat{p}_{k}
\end{gathered}
$$

Optimization problem (Equation 9) determines the amount of power allocation to each stream, which is designated to the $k$-th user node.

Lemma 2. For $2 \times 2$ correlation matrix $\mathbf{R}=[1, \rho ; \rho, 1]$ $(0 \leq \rho \leq 1)$, eigenvalues of $\mathbf{R}$ are $1+\rho$ and $1-\rho$.

Proof 1. From $\operatorname{det}(\mathbf{R}-\lambda \mathbf{I})=0$, we know $\lambda=1-\rho$ or $1+\rho$.

According to Lemma 2 and the observation that the effective channel of Equation 5 is the receive antenna correlation matrix $\mathbf{R}_{r_{k}}$, we have the singular value matrix of $\mathbf{R}_{r_{k}}$ as $\Lambda_{k}=\left[1+\rho_{r_{k}}, 0 ; 0,1-\rho_{r_{k}}\right]$ when each user node has two receive antennas. Replacing $\Lambda_{k}$ in Equation 9 with $\left[1+\rho_{r_{k}}, 0 ; 0,1-\rho_{r_{k}}\right]$, we can derive the closed form solution given by

$$
\begin{aligned}
& \gamma_{k, 1}=\frac{\beta_{k}\left(1-\rho_{r_{k}}\right)\left(1+\rho_{r_{k}}\right)^{2} \hat{p}_{k}+4 \rho_{r_{k}}}{2 \beta_{k}\left(1+\rho_{r_{k}}\right)^{3}\left(1-\rho_{r_{k}}\right)} \\
& \gamma_{k, 2}=\frac{\beta_{k}\left(1-\rho_{r_{k}}\right)\left(1+\rho_{r_{k}}\right)^{2} \hat{p}_{k}-4 \rho_{r_{k}}}{2 \beta_{k}\left(1+\rho_{r_{k}}\right)^{2}\left(1-\rho_{r_{k}}\right)^{2}}
\end{aligned}
$$

where $\gamma_{k, 1}$ and $\gamma_{k, 2}$ are the diagonal elements of $\hat{\Gamma}_{k}$ and $\beta_{k} \triangleq g_{k} \sigma_{n}^{-2}$ (See Appendix for details). Note that when $\rho_{r_{k}}=0$, the equal power is allocated as $\gamma_{k, 1}=\gamma_{k, 2}=\frac{\hat{p}_{k}}{2}$.

In the second step, we can decide the amount of allocated power to each stream without a bisection search. With the proposed two-step method, we can reduce the search space from $K N_{r}$ to $K$. 
Table 1 Simulation parameters

\begin{tabular}{ll}
\hline Cell radius & $\mathbf{1} \mathbf{~ k m}$ \\
\hline Bandwidth & $20 \mathrm{MHz}$ \\
\hline Number of user $K$ & 20 \\
\hline Number of BS antenna $N_{t}$ & $40 \sim 200$ \\
\hline Number of user terminal antenna $N_{r}$ & 2 \\
\hline Maximum transmission power & $32 \mathrm{dBm}$ \\
\hline Distance-dependent path loss & $128.1+37.6 \mathrm{log}(r) \mathrm{dB}, r$ kilometers \\
\hline Receiver noise density & $-101 \mathrm{dBm} / \mathrm{Hz}$ \\
\hline Noise figure & $5 \mathrm{~dB}$ \\
\hline
\end{tabular}

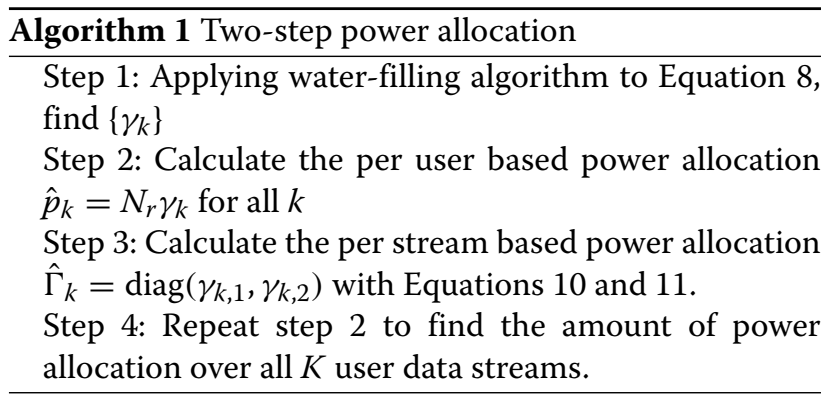

The proposed two-step power allocation method is summarized in Algorithm 1. The resultant two-step precoders are represented as $\mathbf{T}_{k}^{(\mathrm{ts})}=\mathbf{H}_{k}^{H} \mathbf{V}_{k} \hat{\Gamma}_{k}^{1 / 2}, \forall k$. With the proposed two-step method, we can reduce the dimensions of the search space from $K N_{r}$ to $K$.
Note that the generalization of the second step to more than two receive antennas $\left(N_{r}>2\right)$ is straightforward. Instead of closed form solutions, however, it requires numerical search in Step 3 of Algorithm 1 as follows.

Step 3 : Applying water-filling algorithm to Equation 9, calculate the per stream based power allocation $\hat{\Gamma}_{k}$.

\section{Simulation results}

For the simulation of the sum-rate performance, we set $K=20, N_{t}=40 \sim 200, N_{r}=2$, and $\rho_{r_{k}}=0$ and 0.5 for all $k$. Table 1 gives the simulation parameters. The cell radius is $1 \mathrm{~km}$. For the consistency of the simulation, the location of each user is randomly selected from uniform distribution $\mathcal{U}(0,1)$ and is kept fixed over simulation runs. The distance-dependent path loss is $128.1+37.6 \log (r)$ $\mathrm{dB}$ ( $r$ kilometers). The maximum transmission power is set to $32 \mathrm{dBm}^{\mathrm{d}}$. The receiver noise density is set to -101 $\mathrm{dBm} / \mathrm{Hz}$, and the noise figure is $5 \mathrm{~dB}$. The results are averaged over 1,000 independent trials. Figure 1 shows the average sum-rate performance of the optimum power allocation method over the receive antenna correlation $\rho_{r_{k}} \in[0,0.9999]$ for all $k$. We observe that as the receive antenna correlation increases, the sum-rate performance decreases. Specifically, with $N_{t}=200$, when receive correlation is less than or equal to 0.9 , the sum rate drops less than $10 \%$. However, when $\rho_{r_{k}}=0.99$ and $\rho_{r_{k}}=0.9999$, the sum rates drop $21 \%$ and $37 \%$, respectively. Figure 2 shows the average sum-rate performance of the optimum power allocation method in Section 33 and the proposed two-step method in Section 44. For comparison, we plot

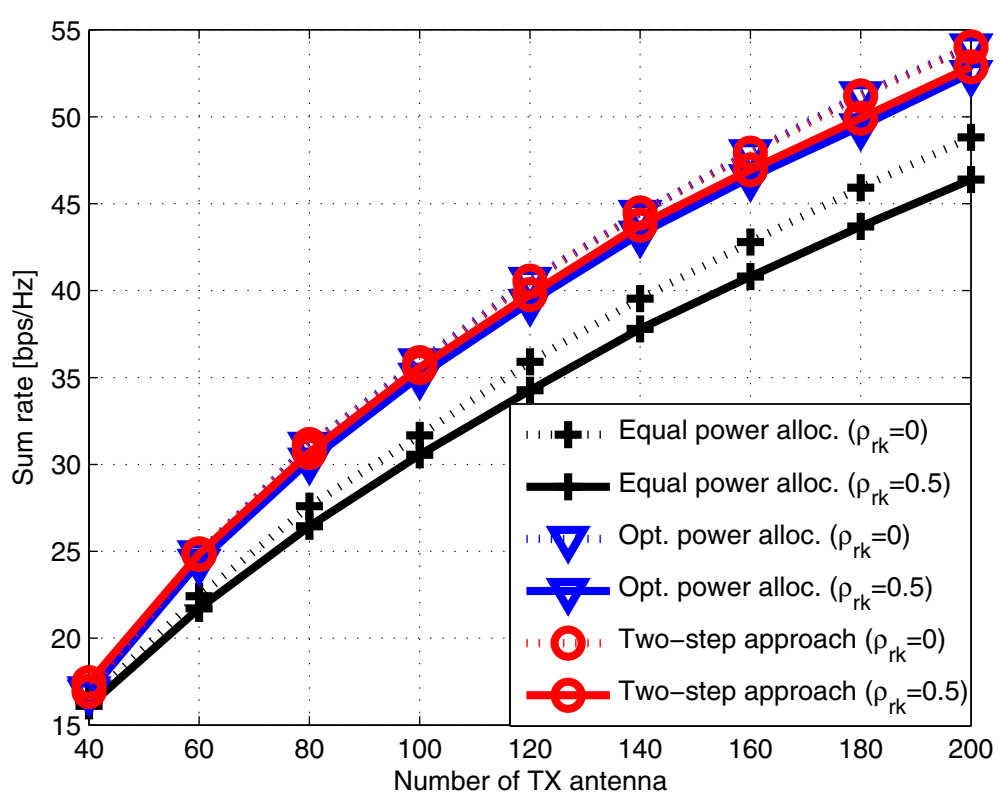

Figure 1 The sum-rate performances over receiver correlation $\rho_{r_{k}}=0 \sim 0.9999$ for all $k$ when $K=20, N_{t}=40 \sim 200$, and $N_{r}=2$. 


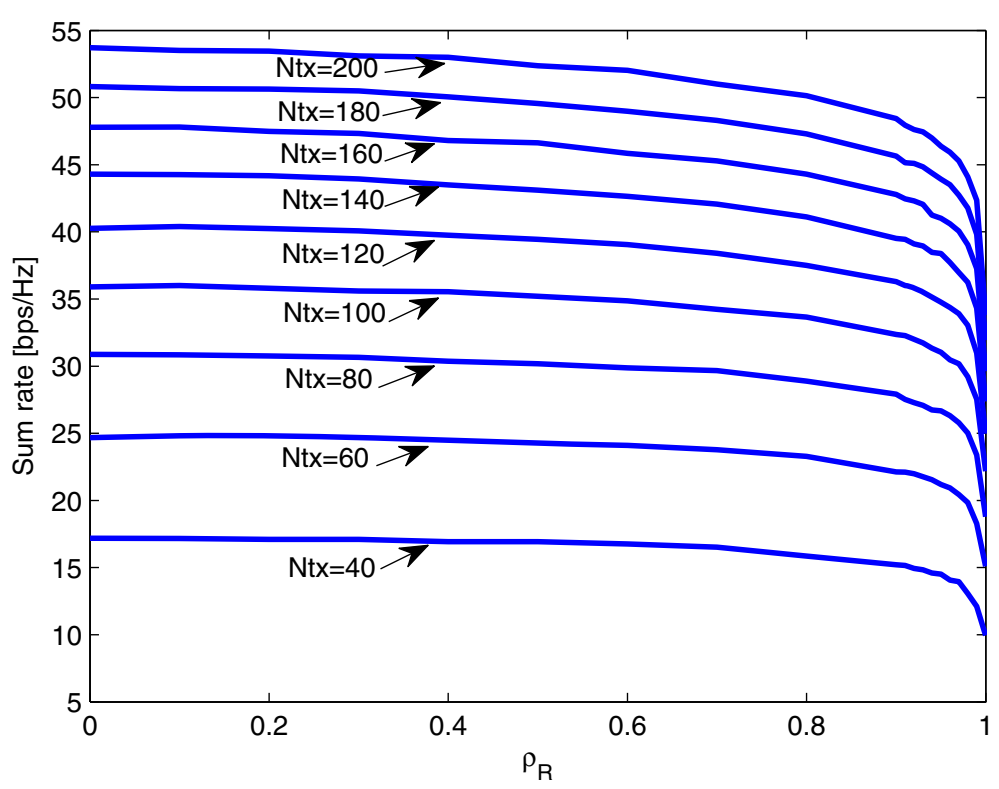

Figure 2 The sum-rate performances when $K=20, N_{t}=40 \sim 200, N_{r}=2$, and $\rho_{r_{k}}=0$ and 0.5 for all $k$.

the equal power allocation method in which the precoder is represented as $\mathbf{T}_{k}^{\mathrm{eq}}=\sqrt{\alpha} \mathbf{H}_{k}^{H} \mathbf{V}_{k}$ for all $k, \alpha=P_{t} / K N_{r}$. Note that the proposed two-step method achieves the optimum sum-rate performance while it can be implemented with low complexity by reducing the search space. In addition, we verify that both proposed methods outperform the equal power allocation method.

\section{Conclusion}

In this paper, we have proposed a MU-MIMO transceiver design method for large-scale antenna systems using the sum-rate maximization criterion when each user node is equipped with multiple receive antennas. Inspired by the favorable propagation characteristics of the large-scale antenna systems, we have proposed a matched-filteringbased optimal transceiver design method that maximizes the sum rate. In addition, we have proposed a suboptimal two-step power allocation method with low computational complexity by reducing the dimension of the bisection search space from $K N_{r}$ to $K$. Through the simulation results, the effectiveness of the proposed transceiver design methods have been validated.

\section{Endnotes}

${ }^{\text {a }}$ This assumption is reasonable because in general the transmit antennas at base station of the large-scale MIMO systems can be deployed to have a large separation among them.

${ }^{\mathrm{b}}$ This is an approximate result assuming $N_{t} \rightarrow \infty$

${ }^{\mathrm{c}}$ Note that current specification of mobile wireless communications supports mobile terminals with two receive antennas [13]
${ }^{\mathrm{d}}$ Considering the power saving effect of large-scale MIMO systems [1], we choose much less transmission power than the ordinary base station transmission power, $43[\mathrm{dBm}]$

\section{Appendix}

Using $\beta_{k} \triangleq g_{k} \sigma_{n}^{-2}$, and $\Gamma_{k}=\operatorname{diag}\left(\gamma_{k, 1}, \gamma_{k, 2}\right)$, the optimization problem (Equation 9) can be rewritten as

$$
\begin{aligned}
& \arg _{\left\{\gamma_{k, 1}, \gamma_{k, 2}\right\}} \max \log \left(1+\beta_{k}\left(1+\rho_{r_{k}}\right)^{2} \gamma_{k, 1}\right) \\
& \quad+\log \left(1+\beta_{k}\left(1-\rho_{r_{k}}\right)^{2} \gamma_{k, 2}\right) \\
& \text { subject to }\left(1+\rho_{r_{k}}\right) \gamma_{k, 1}+\left(1-\rho_{r_{k}}\right) \gamma_{k, 2} \leq \hat{p}_{k}
\end{aligned}
$$

Because there are no inter-stream interferences, the optimum power allocation should satisfy the equality constraint, $\left(1+\rho_{r_{k}}\right) \gamma_{k, 1}+\left(1-\rho_{r_{k}}\right) \gamma_{k, 2}=\hat{p}_{k}$. Then, we have

$$
\gamma_{k, 2}=\frac{\hat{p}_{k}-\left(1+\rho_{r_{k}}\right) \gamma_{k, 1}}{\left(1-\rho_{r_{k}}\right)}
$$

Since this is a convex optimization problem over $\gamma_{k, 1}$, from the Karush-Kuhn-Tucker (KKT) condition [14], the optimum value $\gamma_{k, 1}$ is obtained as

$$
\gamma_{k, 1}^{(\mathrm{opt})}=\frac{\beta_{k}\left(1-\rho_{r_{k}}\right)\left(1+\rho_{r_{k}}\right)^{2} \hat{p}_{k}+4 \rho_{r_{k}}}{2 \beta_{k}\left(1+\rho_{r_{k}}\right)^{3}\left(1-\rho_{r_{k}}\right)}
$$

The parameter $\gamma_{k, 2}^{(\mathrm{opt})}$ can be derived by replacing $\gamma_{k, 1}$ in Equation 6.2 by 6.3 . 


\section{Competing interests}

The authors declare that they have no competing interests.

Received: 6 November 2014 Accepted: 3 February 2015

Published online: 20 March 2015

\section{References}

1. F Rusek, D Persson, BK Lau, EG Larsson, TL Marzetta, O Edfors, F Tufvesson, Scaling up MIMO: opportunities and challenges with very large arrays. IEEE Signal Process. Mag. 30, 40-60 (2013)

2. L Lu, GY Li, AL Swindlehurst, A Ashikhmin, R Zhang, An overview of massive MIMO: benefits and challenges. IEEE J. Select. Topics Signal Process. 8, 742-758 (2014)

3. EG Larsson, O Edfors, F Tufvesson, TL Marzetta, Massive MIMO for next generation wireless systems. IEEE Commun. Mag. 52, 186-195 (2014)

4. HQ Ngo, EG Larsson, TL Marzetta, Energy and spectral efficiency of very large multiuser MIMO systems. IEEE Trans. Commun. 8, 1436-1449 (2013)

5. TL Marzetta, Noncooperative cellular wireless with unlimited numbers of base station antennas. IEEE Trans. Wireless Commun. 9, 3590-3600 (2010)

6. RC Lamare, Massive MIMO systems: Signal processing challenges and research trends. ArXiv pre-print cs.IT/1310.7282. Available: http://arxiv. org/abs/1310.7282

7. TWild, C Hoek, J Hoydis, ST Brink, in Proc. IEEE Int. Symp. Wireless Commun Systems (ISWCS, Paris). Channel measurements for large antenna arrays, (2012), pp. 811-815

8. X Gao, F Tufvesson, O Edfors, F Rusek, in Proc. Asilomar Conf. Signals, Syst., Comput, Pacific Grove. Measured propagation characteristics for very large MIMO at 2.6 GHz, (2012), pp. 295-299

9. H Yang, TL Marzetta, Performance of conjugate and zero-forcing beamforming in large-scale antenna systems. IEEE J. Select. Areas Commun. 31, 172-179 (2013)

10. J Jose, A Ashikhmin, TL Marzetta, S Vishwanath, Pilot contamination and precoding in multi-cell TDD systems. IEEE Trans. Wireless Commun. 10, 2640-2651 (2011)

11. D Shiu, GJ Foschini, MJ Gans, JM Kahn, Fading correlation and its effect on the capacity of multielement antenna systems. IEEE Trans. Commun. 48, 502-513 (2000)

12. TM Cover, JA Thomas, Elements of Information Theory. (Wiley, 111 River Street Hoboken, NJ, United States, 1991)

13. 3GPP TR 36.211 V 11.1.0 (Release 11): Evolved Universal Terrestrial Radio Access (E-UTRA) (2012). Available: http://www.etsi.org/deliver/etsi_ts/ 136200_136299/136211/11.01.00_60/ts_136211v110100p.pdf

14. S Boyd, L Vandenberghe, Convex Optimization. (Cambridge University Press, University Printing House Shaftesbury Road, Cambridge, United Kingdom, 2004)

\section{Submit your manuscript to a SpringerOpen ${ }^{\mathcal{O}}$ journal and benefit from:}

- Convenient online submission

- Rigorous peer review

- Immediate publication on acceptance

- Open access: articles freely available online

- High visibility within the field

- Retaining the copyright to your article

Submit your next manuscript at springeropen.com 